\title{
BRIDGING THE GAP BETWEEN NEUROIMAGING AND NEURONAL PHYSIOLOGY
}

\author{
DAE-SHIK KIM AND KAMIL UGURBIL \\ Center for Magnetic Resonance Research, University of Minnesota Medical School, MN 55455, USA \\ e-mail: dskim@cmrr.umn.edu \\ (Accepted April 30, 2002)
}

\begin{abstract}
Despite the fact that blood oxygenation level dependent (BOLD) functional magnetic resonance imaging (fMRI) studies have become ubiquitous and are of ever increasing importance for clinical and basic neurosciences, the fundamental relationships between BOLD and the underlying neuronal physiology are not understood. This raises severe concerns about the validity of BOLD contrast per se, and the conceptual frameworks currently employed in interpreting cognitive neuroimaging data. In order to expand the explanatory power of functional MRI data, several crucial questions will have to be addressed. The two most important questions are: First, what is the ultimate spatial resolution of fMRI? And secondly, what is the "heural correlate" of functional MRI? This article attempts to compile a series of results from our and other laboratories, suggesting that both the questions of "spatial specificity" and "heural correlate" might be within the reach of a tentative solution, thus finally bridging the gap between functional neuroimaging and neuronal physiology.
\end{abstract}

Keywords: BOLD specificity, cat visual cortex, fMRI, neural correlate.

\section{INTRODUCTION}

One of the foundations of modern neuroscience is the concept of spatial clustering of cortical function. Neurons with similar response properties, such as ocular dominance and orientation preference in the visual cortex of cats, are clustered into iso-functional domains, forming cortical "columns" and "maps" in the vertical and tangential directions to the cortical plate, respectively. The structure, development and plasticity of cortical columns and maps have been studied extensively using a variety of techniques in the past (Payne and Peters, 2002). Each of the traditional mapping techniques, however, suffers from a fundamental limitation. While single and multiunit recording provide the gold standard for defining neuronal receptive field properties, it is very difficult to use these techniques to study the overall layout of the receptive field properties in a given area. This problem can be partly overcome by using histological activity labels such as 2-deoxyglucose (Sokoloff et al., 1977). However, this method cannot be used for in vivo studies, as the experimental animal has to be sacrificed before the radioactive labeling pattern can be visualized. Furthermore, this method allows the cortical pattern of activity in response to a maximum of 2 different stimuli to be labeled, and this significantly limits the number of independent map properties that can be studied (Lowel et al., 1987). The more recently developed "optical imaging of intrinsic signals" allows the simultaneous recording of neuronal activity over large areas of cortex (Frostig et al., 1990). Utilizing the fact that gray matter tissues dominated by deoxyhemoglobin absorb relatively more light at near-infrared wavelength $(605 \pm 10 \mathrm{~nm})$, one can discern the "active" areas of the cortex by shining light on the exposed cortical surface. The ability of optical imaging to resolve individual columns (Bonhoeffer and Grinvald, 1991) is assumed to be based on the facts that a) individual neurons in mammalian cortex are never more than 20-30 $\mu \mathrm{m}$ away from a microcapillary (Pawlik et al., 1981), and b) mammalian neurons are spatially grouped according to their functional specificity. While the optical imaging technique has been extensively utilized to examine the tangential organization of cortical maps (Hubener et al., 1997; Kim et al., 1999), unfortunately, this technique provides only very limited depth information, because only signals from the superficial 300-500 $\mu \mathrm{m}$ can be accessed (Yae et al., 1992; Bonhoeffer and Grinvald, 1993; Stetter and Obermayer, 1999; Stetter et al., 2000). Furthermore, optical imaging at this spatial resolution 
is an invasive technique, since a craniotomy has to be performed to expose the cortical surface. A routine use of optical imaging for assessing human cognitive functions is therefore not possible at this point except with a very coarse spatial resolution which is unsuitable for cognitive studies.

The rapid progress of blood oxygenation level dependent (BOLD) functional magnetic resonance imaging (fMRI) in recent years (Bandettini et al., 1992; Kwong et al., 1992; Ogawa et al., 1992) has raised the hope that the neural bases of cognitive functions can be precisely localized in the brain. Unlike the aforementioned existing neuroimaging techniques, the non-invasive nature of MRI makes it ideally suited for studying functional architecture of human and animal brains, enabling functional homologies between human and animal brains to be suggested. The BOLD technique is based on the use of deoxyhemoglobin as nature's own intravascular paramagnetic contrast agent (Pauling and Coryell, 1936; Thulborn et al., 1982; Ogawa et al., 1990). When placed in a magnetic field, deoxyhemoglobin alters the magnetic field in its vicinity, particularly when it is compartmentalized as it is within red blood cells and the vasculature. The effect increases as the concentration of deoxyhemoglobin increases. At concentrations found in venous blood, a detectable local distortion of the magnetic field surrounding red blood cells and the surrounding blood vessel is produced. This affects the magnetic resonance behavior of the water proton nuclei within and surrounding the vessels, which in turn results in decreases in the transverse relaxation times $\left(\mathrm{T}_{2}\right.$ and $\mathrm{T}_{2}{ }^{*}$; (Thulborn et al., 1982; Ogawa et al., 1990). During activation of the brain, this process is reduced; an increase in neuronal and metabolic activity results in a reduction of the relative deoxyhemoglobin concentration due to an increase of blood flow (and hence increased supply of fresh oxyhemoglobin) that follows. Consequently, in conventional BOLD fMRI, brain activity can be measured as an increase in $\mathrm{T}_{2}$ or $\mathrm{T}_{2} *$ weighted $\mathrm{MR}$ signals (Bandettini et al., 1992; Kwong et al., 1992; Ogawa et al., 1992).

\section{FUNDAMENTAL SPATIAL SPECIFICITY OF BOLD FMRI}

Since its introduction about 10 years ago, BOLD fMRI has been successfully applied, among numerous other examples, to precisely localize correlates of cognitive (Wagner et al., 1998), motor (Kim et al., 1993), and perceptual (Sereno et al., 1995; Engel et al., 1997; Tootell et al., 1997) functions in the human cerebral cortex. While such functional brain imaging studies have provided unprecedented amount of insights into the workings of the human brain in vivo, almost all BOLD studies in humans are performed today at the spatial scale of centimeters to several millimeters. Localization information at this coarse scale is of pivotal relevance for questions originating from cognitive psychology and cognitive neurosciences. The ability of BOLD fMRI to map the details of the brain's functional architecture at the neurophysiologically more relevant, millimeter and sub-millimeter scale, however, remains unknown.

Interestingly, recent studies from several labs suggest that there might be an intriguing relationship between the fundamental spatial resolution of BOLD and its underlying hemodynamic processes. This is due to the fact that the BOLD signal is not a direct measure of neuronal activity per se. Rather, it is a complex convolution of changes in cerebral hemodynamic balance following a focal neural activity. Recent optical spectroscopy and functional MRI data (Malonek and Grinvald, 1996; Malonek and Grinvald, 1997; Vanzetta and Grinvald, 1999; Kim et al., 2000a) indicate that the spatial specificity of BOLD could be further and more dramatically improved if an initial decrease of MR signals can be utilized for functional imaging formation. The proposed hypothesis (see (Kim et al., 2002) for details) here is that the first event following focal neuronal activity will be an increase in oxygen consumption, caused by an elevation in oxidative metabolism of active neurons. Based on 2-DG data (Sokoloff et al., 1977), we can assume the increase in oxidative metabolism in mammalian cortex to be colocalized with the site of electrical activity. The increase in oxidative metabolism will naturally elevate the local deoxyhemoglobin content in the parenchyma of active neurons, assuming there is no immediate commensurate change in cerebral blood flow (Fox and Raichle, 1986). In $\mathrm{T}_{2}$ or $\mathrm{T}_{2}{ }^{*}$ weighted BOLD fMRI images, such increase in paramagnetic deoxyhemoglobin should therefore be detectable as a decrease in observable MR signals. Such an initial deoxygenation of the local cortical tissue will last only for a short time, as fresh blood (fresh oxyhemoglobin) will rush into capillaries in response to the increased metabolism. Such increase in "fresh" oxyhemoglobin can be assumed to reverse the local ratio of hemoglobin in favor of oxyhemoglobin, thus resulting in an increase in observable MR signals. This delayed oxygenation of the cortical tissue is the basis for conventional BOLD signals (Ogawa et al., 1992). 
Several lines of recent optical spectroscopy studies (Malonek and Grinvald, 1996; Malonek et al., 1997; Vanzetta and Grinvald, 1999; Vanzetta and Grinvald, 2001) suggest that, following sensory stimulation, such a "biphasic" response indeed takes place. These results indicate that the local deoxyhemoglobin concentration increases immediately following stimulation, yielding the maximum concentration about 2-3 seconds after stimulus onset. Oxygen consumption increase appears to be, at least in part if not entirely, responsible for this early deoxyhemoglobin increase (Vanzetta and Grinvald, 1999). The local deoxyhemoglobin concentration is subsequently reversed, ultimately resulting in a relatively large decrease in overall deoxyhemoglobin content. The crucial question here is the spatial specificty of the above described "biphasic" hemodynamic processes. To this end, it has been proposed that the two distinct events (initial deoxygenation followed by the delayed oxygenation) ought to result in fundamentally different functional specificity (Malonek and Grinvald, 1996). It is assumed that the initial deoxygenation, reflecting an increase in oxidative metabolism prior to the onset of blood flow enhancement, should be co-registered with the site of electrical activity up to the level of individual cortical columns. The oxygen consumption occurs in the mitochondria contained in the cells of the tissue. The consequent alterations in deoxyhemoglobin will therefore occur in the capillaries located in this tissue initially, before propagating down the venous system due to blood flow. The well-established "optical imaging of intrinsic signals"(Grinvald et al., 1986; Frostig et al., 1990), which has been crossvalidated with single unit techniques (Shmuel and Grinvald, 1996; Antonini et al., 1998; Crair et al., 1998), is similarly based on measuring the local transient increase of deoxyhemoglobin. The delayed oxygenation of the cortical tissue, on the other hand, is suggested to be far less specific. The assumption here is that the "fidelity" of the cortical vasculature is not sufficient in order to provide fresh oxyhemoglobin selectively to the active columns only. Instead, the entire local vicinity of the active column is flooded with fresh blood. Consequently, such delayed oxygenation signals, as detected with conventional BOLD technique, may surpass the spatial extent of the actual activated area by several millimeters (Malonek and Grinvald, 1996; Malonek et al., 1997).

Both the existence of "biphasic" BOLD response per se, and the suggested differences in functional specificity has been the subject of heated controversies in recent years. While the initial deoxygenation signal in fMRI (termed 'initial dip") has been reported in awaking behaving humans (Menon et al., 1995; Hu et al., 1997) and anesthetized monkeys (Logothetis et al., 1999), studies in rodents failed to detect any significant initial decrease in BOLD signal following sensory stimulation (Marota et al., 1999; Silva and Kim, 1999). However, more recent studies suggest that the hemodynamic regulation of the rodents might pose rather an exception among the animal species, since a slight variation in experimental design and/or analysis methods yielded vastly conflicting results (see (Buxton, 2001; Mayhew et al., 2001; Vanzetta and Grinvald, 2001) for discussion). It appears reasonable to assume that the "dip" as a hemodynamic process does exist, while its detection through MRI may pose practical problems due to its small signal amplitude $(\sim 0.5 \%)$ and susceptibility to physiological noises.

The question of whether use of the initial dip would indeed improve the spatial specificity of BOLD has been far more difficult to address experimentally. This is largely because most fMRI studies examining this phenomenon so far has been conducted in humans, therefore, by necessity using relatively coarse nominal spatial resolution above the level of the individual cortical columns. However, in a recent study, pre-operative functional MRI and intraoperative optical imaging were performed in the same human subject. (Cannestra et al., 2001). While the spatial overlap between optical imaging and conventional (positive) fMRI was poor, there was a dramatic improvement in spatial correspondence between the two dataset when the initial dip portion of the MRI signal was used.

A more direct case in support of the improved spatial specificity of the initial dip was provided in a recent series of studies by our group (Duong et al., 2000; Kim et al., 2000a; Kim et al., 2000b) in anesthetized cat primary visual cortex. Here, consistent with awake human and anesthetized primate data, the time evolution of MR signals was found to be clearly biphasic: following visual stimulation, the MR signal decreases, reaching a minimum of about $-0.4 \%$ around 3 seconds after the stimulus onset. The signal then reversed, yielding a maximum positive signal change of $1.0 \sim 2.0 \%$ about $8 \sim 10$ seconds following stimulus onset. Furthermore, the two functional maps displayed based on the "Initial negative" and the "delayed positive" signals yielded a fundamentally different pattern of activity in response to identical visual stimulus (Kim et al., 2000a). Unlike in the "positive" map, the foci of the negative signal changes were confined to "patchy" clusters. In line with the iso-orientation columns in cat primary visual 
cortex, observed using 2-DG (Lowel et al., 1987) and optical imaging (Bonhoeffer and Grinvald, 1991; Kim and Bonhoeffer, 1994; Kim et al., 1999) techniques, such semi-ellipsoidal and irregularly shaped clusters were distributed all over the approximated area 18 with an average periodicity of about $1.34 \pm 0.23 \mathrm{~mm}$. The crucial question here was whether those "column-like" domains represent indeed genuine iso-orientation domains. To this end, we took advantage of the fact that the spatial layout of the orientation columns in cat primary visual cortex displays a set of characteristic topological features. For example, it is well known that the orientation domains for orthogonal orientations are complementary to each other. The results of our complementarity tests clearly demonstrated that each pattern of "hegative" activity is highly specific to the respective stimulus orientation: patches for orthogonal orientations occupy cortical territories that are mostly complementary to each other. Finally, as a further way of indirect validation, we attempted to derive the distinct topology of the composite orientation map in cat primary visual cortex using our negative BOLD maps. A characteristic and invariant feature of the mammalian orientation system is the existence of "topological singularities" that were observed across many mammalian species using both multi-electrode (Swindale et al., 1987) and optical imaging (Bonhoeffer and Grinvald, 1991) techniques. Using our negative BOLD fMRI data, composite angle maps for the negative BOLD changes were obtained through a pixel-by-pixel vector addition of the four iso-orientation maps with the negative percent changes as vector amplitudes and the respective stimulus orientations as vector angles. In such composite maps based on the early negative BOLD signals, the preferred orientations changed smoothly, forming a "map" of orientation selectivity. The continuity of orientation preferences was interrupted only at the singular points (termed orientation "pinwheels") where the domains for all orientations converge. Each orientation was found to be represented only once around such a "pinwheel", thereby giving rise to two types of topological singularities according to their rotational chiralities. The pinwheel density and the ratio between "clockwise" and "counter-clockwise" singularities found in negative BOLD composite maps were in excellent agreement with those obtained with optical imaging techniques. In our study, a pinwheel density of $1.46 \pm 0.17$ pinwheels $/ \mathrm{mm}^{2}$ was found, while optical imaging studies (Bonhoeffer and Grinvald, 1991; Kim and Bonhoeffer, 1994) yielded average pinwheel densities between 1.2 1.95 pinwheels $/ \mathrm{mm}^{2}$.
Likewise, the ratio between clockwise and counterclockwise pinwheels was found to be roughly 1:1 in both negative-BOLD and optical imaging data.

As previously discussed, based on optical imaging studies, the stimulus-evoked delayed hyperoxygenation state was reported to be spatially diffuse and less suited for resolving the columnar layouts (Malonek and Grinvald, 1996). However, optical imaging with intrinsic signals does not provide a direct measure of cerebral blood flow (CBF). Since MR based methods can in fact obtain CBF based maps directly, the specificity of the CBF response at the tissue level can be evaluated directly using CBF based fMRI. Though less widely used than the BOLD technique because of its relatively poor temporal resolution (on the order of $1-6 \mathrm{~s}$ in comparison to sub-second resolution for BOLD), CBF-based fMRI can be targeted to map flow into the capillaries and the tissue (due to exchange across capillary walls), avoiding large draining vein contributions. Using the flow-sensitive alternating inversion recovery (FAIR) fMRI technique (Kim, 1995; Kim and Tsekos, 1997; Kim and Ugurbil, 1997) for imaging CBF changes at the tissue level (i.e. imaging tissue perfusion), the point spread function of perfusion increases under a singlestimulus condition was recently investigated in the cat visual cortex (Duong et al., 2001). The veracity of the CBF-based single-condition columnar maps was evaluated using the complementarity of orthogonal orientation stimuli. Functional iso-orientation columns responding to two orthogonal stimuli were shown to occupy approximately complementary domains and the "complementarity" between orthogonal maps was statistically significant. A marked CBF increase $(\sim 55 \%)$ following $45^{\circ}$ or $135^{\circ}$ stimulus was observed in each region tuned to one of these orientations, while the stimulation with the orthogonal orientation lead to a significantly smaller CBF increase (on the average $3.3 \pm 0.6$ fold smaller).

\section{RELATIONSHIP BETWEEN BOLD FMRI AND ELECTROPHYSIOLOGY}

The above results clearly suggest that the functional specificity of BOLD at neurophysiologically relevant columnar scale depends highly on the hemodynamic modality of the underlying signals (Kim et al., 2000a; Duong et al., 2001). If the early negative signals or CBF contrast are utilized, functional maps at "columnar resolution" can be obtained. The delayed positive conventional BOLD changes on the other hand, were clearly indicative for the pattern of the overall activation per se in the visual cortex, but were less suited to discriminate between the active and inactive 
columns, since they were more diffuse and were less specific for the individual stimulus properties.

While such results are in line with optical imaging studies, and have been furthermore "verified" through geometric and topological considerations (Kim et al., 2000a), it is clear that an ultimate validation of the veracity of the such fMRI based "iso-orientation columns" would require a clear understanding of the coupling between functional MRI and the underlying neuronal events. To this end, it is quite surprising to note that despite the overwhelming prominence of BOLD fMRI for clinical and basic neurosciences, a direct neurophysiological correlate of BOLD responses has never been firmly established. This is particularly unsatisfying, since the BOLD signal is not a direct measure of neuronal activity per se. Rather, it is a complex convolution of changes in cerebral metabolic rate of oxygen $\left(\mathrm{CMRO}_{2}\right)$, cerebral blood flow (CBF) and cerebral blood volume (CBV) following focal neuronal activity. Therefore, a direct and linear correlation between the amplitude of the observable BOLD signals and the underlying neural activity can therefore not be assumed a priori. Unfortunately, the existing evidences on the coupling between the BOLD fMRI signal and the underlying neuronal physiology are both sparse and conflicting. Furthermore, it is not always clear, what we mean by asking to "bridge the gap between neuroimaging and neurophysiology". What is the essence of the question of the "heural correlate of BOLD'? Based on pragmatic criteria, we suggest the following four questions to be most significant:

Question 1: Do BOLD signal changes reflect the magnitude of neural activity change linearly? This is the most fundamental of all questions. If we observe BOLD contrasts of $\alpha$ and $\alpha+\Delta$ for two disjoint stimulus conditions, respectively, where $\alpha$ is the BOLD contrast, and $\Delta$ a constant, can we assume the underlying neuronal signals to be $\alpha^{\prime}$ and $\alpha^{\prime}+\Delta^{\prime}$ ? A small, but increasing body of results seems to indicate a predominantly linear coupling between BOLD and neuronal activity. For example, cross-species comparison between single unit data obtained in primates and fMRI data in humans during similar perceptual tasks suggested that fMRI responses might be proportional to the underlying firing rate of the neurons (Heeger et al., 2000; Rees et al., 2000). Such a linear relationship was also observed in anesthetized monkeys by Logothetis et al. (2001) in a recent technical tour de force, in which the single unit responses were acquired together with BOLD fMRI signals inside the MRI scanner. Although comforting for the existing body of cognitive neuroimaging data, this is rather surprising, as there is a great deal of potential non-linearity between hemodynamic and neuronal processes. Such non-linearity is particularly evident in the temporal domain: Neuronal activity occurs in the order of milliseconds, while the hemodynamic responses can take up to several seconds to reach its maximum to the same stimulus. Indeed, when large scale neuronal responses, as measured with visual evoked potentials (VEP) where directly compared with functional MRI signals in human visual cortex (Janz et al., 2001), it was found that the time course of the BOLD fMRI cannot be adequately predicted by the amplitude of the VEP signals. Moreover, it is not clear over which spatial scale the linearity between BOLD and neuronal responses (if at all) holds true. Is it really invariant across the different spatial scales of the cortical architecture? Can we assume a universal linearity from the scale of entire cortical areas (several millimeters to centimeters) to individual columns (sub-millimeter)? This question was addressed in a recent series of experiments in our lab (Kim et al., 2001). In these studies, conventional BOLD fMRI in cat primary visual cortex was observed first. The amplitude and spatial location of the BOLD signals were then correlated with single/multi unit recording data acquired subsequently outside the magnet. A proper alignment between the BOLD and electrophysiological data was achieved by utilizing an implanted agar grid that was first imaged and then later employed as a Cartesian guide for placing electrodes outside the MRI scanner (Kim et al., 2000c; Toth et al., 2001). The results of our studies suggest that there is a robust linear correlations between BOLD and single unit measures if BOLDsingle unit pairs from a sufficient large area are averaged. The average correlation between the two responses was found to be around $\mathrm{R}^{2}=0.7$. However, when the BOLD and single unit measures were compared at the level of individual recording sites the correlations between BOLD and single unit responses broke down rapidly (Toth et al., 2001).

Question 2: Do BOLD signal changes reflect single cell or population responses? Given the nominal voxel size of most fMRI scans (several millimeters at best), it is trivial to conclude that BOLD reflects the activity of many neurons (let's say, for a voxel of 1$2 \mathrm{~mm}^{3}$ around $10^{5}$ neurons (Braitenberg, 2001)). The overwhelming body of existing electrophysiological data, however, is based on electrode recordings from single (single-unit recording, SUA) or a handful of neurons (multi-unit recording, MUA). The real question 
is hence to ask how accurately the responses of single cells (our "gold standard" given the existing body of data) are reflected by a population response, such as in BOLD fMRI. Theoretically, if each neuron would "fire" independently of its neighbor's behavior, this would be an ill-posed problem, as fMRI will not be able to distinguish small activity changes in a large cellular population from large changes in a small population. Fortunately however, neurons are embedded in tight local circuitries, forming functional clusters with similar receptive field properties raging from "microcolumns", "columns", to "hyper-columns". Both the neuronal firing rate and phase are correlated between neighboring neurons (Singer, 1999), and in most sensory areas there is a good correlation between local field potentials (LFP), which are assumed to reflect the average activity of a large number of neurons, and the responses of individual spiking neurons. In fact, it is difficult to imagine how BOLD contrast could be detectable at all, if it were sensitized to the behavior of uncorrelated individual neurons, as the metabolic demand of a single neuron would be hardly sufficient to initiate the chain of hemodynamic events giving rise to BOLD.

Question 3: Do BOLD signal changes reflect spiking suprathreshold (output) or synaptic subthreshold (input) activity? According to the standard "integrate-and-fire" model of neurons an action potential is generated when the membrane potential reaches threshold by depolarization, which in turn is determined by the integration of incoming excitatory (EPSP) and inhibitory (IPSP) post-synaptic potentials. Action potentials are usually generated only around the axon hillock, while synaptic potentials can be generated all across the dendritic tree (mostly on dendritic spines) and cell soma. The thresholddependent action potential firing means that much more sub- and suprathreshold synaptic activity than action potential activity is likely at any one time. And the much larger neural surface area associated with synaptic activity means that the total metabolic demand (i.e. number of $\mathrm{Na}^{+} / \mathrm{K}^{+}$pumps involved etc.) for synaptic activity ought to be significantly higher than those required for generating action potentials. It seems therefore likely to be the case that BOLD contrast - like other methods based on cortical metabolism, such as 2DG and optical imaging - is dominated by the synaptic subthreshold activity. However, the precise contributions of synaptic and spiking activities are hard to quantify, since with conventional stimuli one would expect synaptic input and spiking output activity to be roughly correlated with each other (Jagadeesh et al., 1993; Ferster, 1994;
Nelson et al., 1994). Indeed, it is not trivial to imagine an experiment where input and output activities would not correlate with each other. One way this has been proposed in the past, is to look in a visual area at spatial activity resulting from the edge of a visual stimulus (Gulyas et al., 1987; Knierim and van Essen, 1992; Grinvald et al., 1994). Since 'extra-classical' receptive fields across such an edge are by definition subthreshold activity, it follows that a stimulus with an edge in it creates regions of cortex where activity is only subthreshold in origin. Existing optical imaging studies (Grinvald et al., 1994; Toth et al., 1996) have concluded that subthreshold activity does indeed contribute significantly to the optical signal, suggesting that it might contribute to the BOLD signal as well.

Question 4: What about inhibitory activity? To the best of our knowledge, there is currently no evidence that the metabolic demands differ greatly between excitatory and inhibitory synapses. Therefore, fundamentally, both the excitatory (EPSP) and inhibitory (IPSP) synaptic inputs can be expected to cause similar metabolic and hemodynamic events ultimately giving rise to similar BOLD contrasts. On the site of the spiking output activity, however, they have an opposite effect: accumulation of EPSPs will increase the probability for spike generation (and therefore also the metabolic demand), while IPSPs will decrease it. Assuming that the BOLD response predominantly reflects changes in synaptic subthreshold activity, it remains elusive whether excitatory and inhibitory cortical events can be differentiated using the BOLD response in any single region. Recently, one group proposed that inhibition, unlike excitation, elicits no measurable change in the BOLD signal (Waldvogel et al., 2000). They hypothesized that because of the lower number of inhibitory synapses (Beaulieu and Colonnier, 1985), their strategically superior location (inhibitory receptors: basal cell body; excitatory receptors: distal dendrites), and increased efficiency (Koos and Tepper, 1999) there could be lower metabolic demand during inhibition compared to excitation. The validity of this claim notwithstanding, both empirical and theoretical studies suggest that excitatory and inhibitory neurons in the cortex are so tightly interconnected in local circuits (Payne and Peters, 2002) for details of the local circuitry in cat primary visual cortex; see also (Kisvarday et al., 1994) for the anatomy of local inhibitory circuits in cats) that one is unlikely to observe an increase in excitation without an increase in inhibition. After all, for an inhibitory neuron to increase its firing rate, it must be receiving more 
excitatory input, and most of the excitatory input comes from the local cortical neighborhood (see Sherpherd, 1990; Payne and Peters, 2002; for overview). Naturally, excitation and inhibition would not occur in temporal unison, as otherwise no cell would reach threshold. On the temporal scale of several hundred milliseconds to seconds during which BOLD contrast emerges (Ogawa et al., 1992), however, such potential temporal differences would most likely be rendered indistinguishable. One viable hypothesis is therefore that BOLD contrast reflects a steady-state balance of local excitation and inhibition. In particular if BOLD is more sensitive to subthreshold than to spiking activity.

\section{CONCLUSIONS}

In less than 10 years since its introduction, functional brain imaging based on BOLD contrast has been firmly established as a gold standard technique for questions in clinical and cognitive neurosciences. Here, at the spatial scale of centimeters to several millimeters, it provided unprecedented insights into the workings of the living brain in a completely non-invasive manner. Utilizing the very same mechanisms for elucidation of the questions at the neurophysiologically more relevant, sub-millimeter, columnar scale will necessitate a much clearer understanding of both the basic hemodynamic origins of the BOLD contrast, and its relationship to the underlying neuro-electrophysiological events. Furthermore, such fMRI at columnar resolution seem to call for modification of the original BOLD contrast (Kim et al., 2000a; Duong et al., 2001).

In conclusion, the studies reviewed in this article suggest, however, that both the questions of "spatial specificity" and "neural correlate" might be within the reach of a tentative solution, thus suggesting that we might be just few steps before a true "neurophysiological” functional MRI.

\section{ACKNOWLEDGEMENTS}

I would like thank Drs T. Duong, S-G Kim, I. Ronen, H. Merkle, and L.J. Toth for their support during the experimental works. This work was funded by the NIH (RR08079; MH61937), The Keck Foundation, The Minnesota Medical Foundation, NARSAD, and The Whitaker Foundation.

\section{REFERENCES}

Antonini A, Gillespie DC, Crair MC, Stryker MP (1998). Morphology of single geniculocortical afferents and functional recovery of the visual cortex after reverse monocular deprivation in the kitten. $\mathbf{J}$ Neurosci 18:9896-909.

Bandettini PA, Wong EC, Hinks RS, Tikofsky RS, Hyde JS (1992). Time course EPI of human brain function during task activation. Magn Reson Med 25:390-7.

Beaulieu C, Colonnier M (1985). A laminar analysis of the number of round-asymmetrical and flat-symmetrical synapses on spines, dendritic trunks, and cell bodies in area 17 of the cat. J Comp Neurol 231:180-9.

Bonhoeffer T, Grinvald A (1991). Iso-orientation domains in cat visual cortex are arranged in pinwheel-like patterns. Nature 353:429-31.

Bonhoeffer T, Grinvald A (1993). The layout of isoorientation domains in area 18 of cat visual cortex: optical imaging reveals a pinwheel-like organization. $\mathrm{J}$ Neurosci 13:4157-80.

Braitenberg V (2001). Brain size and number of neurons: an exercise in synthetic neuroanatomy. J Comput Neurosci 10:71-7.

Buxton RB (2001). The elusive initial dip. Neuroimage 13:953-8.

Cannestra AF, Pouratian N, Bookheimer SY, Martin NA, Becker DP, Toga A (2001). Temporal spatial differences observed by functional MRI and human intraoperative optical imaging. Cereb Cortex, 11.

Crair MC, Gillespie DC, Stryker MP (1998). The role of visual experience in the development of columns in cat visual cortex. Science 279:566-70.

Duong TQ, Kim DS, Ugurbil K, Kim SG (2000). Spatiotemporal dynamics of the BOLD fMRI signals: toward mapping submillimeter cortical columns using the early negative response. Magn Reson Med 44:23142.

Duong TQ, Kim DS, Ugurbil K, Kim SG (2001). Localized cerebral blood flow response at submillimeter columnar resolution. Proc Natl Acad Sci USA 98:10904-9.

Engel SA, Glover GH, Wandell BA (1997). Retinotopic organization in human visual cortex and the spatial precision of functional MRI. Cereb Cortex 7:181-92.

Ferster D (1994). Linearity of synaptic interactions in the assembly of receptive fields in cat visual cortex. Curr Opin Neurobiol 4:563-8.

Fox PT, Raichle ME (1986). Focal physiological uncoupling of cerebral blood flow and oxidative metabolism during somatosensory stimulation in human subjects. Proc Natl Acad Sci USA 83:1140-4.

Frostig RD, Lieke EE, Ts'o DY, Grinvald A (1990). Cortical functional architecture and local coupling between neuronal activity and the microcirculation revealed by in vivo high-resolution optical imaging of intrinsic signals. Proc Natl Acad Sci USA 87:6082-6.

Grinvald A, Lieke E, Frostig RD, Gilbert CD, Wiesel TN (1986). Functional architecture of cortex revealed by optical imaging of intrinsic signals. Nature 324:361-4. 
Grinvald A, Lieke EE, Frostig RD, Hildesheim R (1994). Cortical point-spread function and long-range lateral interactions revealed by real-time optical imaging of macaque monkey primary visual cortex. J Neurosci $14: 2545-68$.

Gulyas B, Orban GA, Duysens J, Maes H (1987). The suppressive influence of moving textured backgrounds on responses of cat striate neurons to moving bars. J Neurophysiol 57:1767-91.

Heeger DJ, Huk AC, Geisler WS, Albrecht DG (2000). Spikes versus BOLD: what does neuroimaging tell us about neuronal activity? Nat Neurosci 3:631-3.

Hu X, Le TH, Ugurbil K (1997). Evaluation of the early response in fMRI in individual subjects using short stimulus duration. Magn Reson Med 37:877-84.

Hubener M, Shoham D, Grinvald A, Bonhoeffer T (1997). Spatial relationships among three columnar systems in cat area 17. J Neurosci 17:9270-84.

Jagadeesh B, Wheat HS, Ferster D (1993). Linearity of summation of synaptic potentials underlying direction selectivity in simple cells of the cat visual cortex. Science 262:1901-4.

Janz C, Heinrich SP, Kornmayer J, Bach M, Hennig J (2001). Coupling of neural activity and BOLD fMRI response: new insights by combination of fMRI and VEP experiments in transition from single events to continuous stimulation. Magn Reson Med 46:482-6.

Kim DS, Bonhoeffer T (1994). Reverse occlusion leads to a precise restoration of orientation preference maps in visual cortex. Nature 370:370-2.

Kim DS, Duong TQ, Kim SG (2000a). High-resolution mapping of iso-orientation columns by fMRI. Nat Neurosci 3:164-9.

Kim DS, Duong TQ, Kim SG (2000b). Reply to "Can current fMRI techniques reveal the micro-architecture of cortex?" Nat Neurosci 3:414.

Kim DS, Duong TQ, Ugurbil K, Kim SG (2002). Functional mapping in the cat primary visual cortex using high magnetic fields. In: Payne BR, Peters A, eds: The cat primary visual cortex. New York: Academic Press.

Kim DS, Matsuda Y, Ohki K, Ajima A, Tanaka S (1999). Geometrical and topological relationships between multiple functional maps in cat primary visual cortex. Neuroreport 10:2515-22.

Kim DS, Ronen I, Duong TQ, Ugurbil K, Kim SG (2000c). Neural correlate of blood oxygenation level dependent functional MRI. Soc Neurosci Abstr, pp. 309.6.

Kim DS, Ronen I, Toth L, Olman C, Duong TQ, Merkle H, Kim SG, Ugurbil K (2001). Neural correlate of BOLD functional MRI. ISMRM.

Kim SG (1995). Quantification of relative cerebral blood flow change by flow-sensitive alternating inversion recovery (FAIR) technique: application to functional mapping. Magn Reson Annu 34:293-301.
Kim SG, Ashe J, Hendrich K, Ellermann JM, Merkle H, Ugurbil K, Georgopoulos AP (1993). Functional magnetic resonance imaging of motor cortex: hemispheric asymmetry and handedness. Science 261: 615-7.

Kim SG, Tsekos NV (1997). Perfusion imaging by a flowsensitive alternating inversion recovery (FAIR) technique: application to functional brain imaging. Magn Reson Med 37:425-35.

Kim SG, Ugurbil K (1997). Comparison of blood oxygenation and cerebral blood flow effects in fMRI: estimation of relative oxygen consumption change. Magn Reson Med 38:59-65.

Kisvarday ZF, Kim DS, Eysel UT, Bonhoeffer T (1994). Relationship between lateral inhibitory connections and the topography of the orientation map in cat visual cortex. Eur J Neurosci 6:1619-32.

Knierim JJ, van Essen DC (1992). Neuronal responses to static texture patterns in area V1 of the alert macaque monkey. J Neurophysiol 67:961-80.

Koos T, Tepper JM (1999). Inhibitory control of neostriatal projection neurons by GABAergic interneurons. Nat Neurosci 2:467-72.

Kwong KK, Belliveau J, Chesler DA, Goldberg IE, Weisskoff RM, Poncelet BP, Kennedy DN, Hoppel BE, Cohen MS, Turner R, Cheng H, Brady TJ, Rosen BR (1992). Dynamic magnetic resonance imaging of human brain acrivity during primary sensory stimulation. Proc Natl Acad Sci USA 89:5675-9.

Logothetis N, Pauls J, Augath M, Trinath T, Oeltermann A (2001). Neurophysiological investigation of the basis of the fMRI signal. Nature 412:150-7.

Logothetis NK, Guggenberger H, Peled S, Pauls J (1999). Functional imaging of the monkey brain. Nat Neurosci 2:555-62.

Lowel S, Freeman B, Singer W (1987). Topographic organization of the orientation column system in large flat-mounts of the cat visual cortex: a 2-deoxyglucose study. J Comp Neurol 255:401-15.

Malonek D, Dirnagl U, Lindauer U, Yamada K, Kanno I, Grinvald A (1997). Vascular imprints of neuronal activity: relationships between the dynamics of cortical blood flow, oxygenation, and volume changes following sensory stimulation. Proc Natl Acad Sci USA 94:14826-31.

Malonek D, Grinvald A (1996). Interactions between electrical activity and cortical microcirculation revealed by imaging spectroscopy: implications for functional brain mapping. Science 272:551-4.

Malonek D, Grinvald A (1997). Vascular regulation at sub millimeter range. Sources of intrinsic signals for high resolution optical imaging. Adv Exp Med Biol 413:215-20.

Marota JJ, Ayata C, Moskowitz MA, Weisskoff RM, Rosen BR, Mandeville JB (1999). Investigation of the early response to rat forepaw stimulation. Magn Reson Med 41:247-52. 
Mayhew J, Johnston D, Martindale J, Jones M, Berwick J, Zheng Y (2001). Increased oxygen consumption following activation of brain: theoretical footnotes using spectroscopic data from barrel cortex. Neuroimage 13:975-87.

Menon RS, Ogawa S, Hu X, Strupp JP, Anderson P, Ugurbil K (1995). BOLD based functional MRI at 4 Tesla includes a capillary bed contribution: echoplanar imaging correlates with previous optical imaging using intrinsic signals. Magn Reson Med 33:453-9.

Nelson S, Toth L, Sheth B, Sur M (1994). Orientation selectivity of cortical neurons during intracellular blockade of inhibition. Science 265:774-7.

Ogawa S, Lee TM, Nayak AS, Glynn P (1990). Oxygenation-sensitive contrast in magnetic resonance image of rodent brain at high magnetic fields. Magn Reson Med 14:68-78.

Ogawa S, Tank DW, Menon R, Ellermann JM, Kim SG, Merkle H, Ugurbil K (1992). Intrinsic signal changes accompanying sensory stimulation: functional brain mapping with magnetic resonance imaging. Proc Natl Acad Sci USA 89:5951-5.

Pauling L, Coryell CD (1936). The magnetic properties and structures of hemoglobin, oxyhemoglobin, and carbonmonoxyhemoglobin. Proc Natl Acad Sci USA 22:210-6.

Pawlik G, Rackl A, Bing RJ (1981). Quantitative capillary topography and blood flow in the cerebral cortex of cats: an in vivo microscopic study. Brain Res 208:3558.

Payne BR, Peters A (2002). The cat primary visual cortex. San Diego: Academic Press.

Rees, G., Friston, K., and Koch, C. (2000): A direct quantitative relationship between the functional properties of human and macaque V5. Nat Neurosci 3, 716-23.

Sereno MI, Dale AM, Reppas JB, Kwong KK, Belliveau JW, Brady TJ, Rosen BR, Tootell RB (1995). Borders of multiple visual areas in humans revealed by functional magnetic resonance imaging. Science 268:889-93.

Sherpherd GM (1990). The synaptic organization of the brain. Oxford: Oxford University Press.

Shmuel A, Grinvald A (1996). Functional organization for direction of motion and its relationship to orientation maps in cat area 18. J Neurosci 16:6945-64.

Silva AC, Kim SG (1999). Pseudo-continuous arterial spin labeling technique for measuring CBF dynamics with high temporal resolution. Magn Reson Med 42:425-9.

Singer W (1999). Time as coding space? Curr Opin Neurobiol 9:189-94.
Sokoloff L, Reivich M, Kennedy C, Des Rosiers MH, Patlak CS, Pettigrew KD, Sakurada O, Shinohara M (1977). The [14C]deoxyglucose method for the measurement of local cerebral glucose utilization: theory, procedure, and normal values in the conscious and anesthetized albino rat. J Neurochem 28:897-916.

Stetter M, Obermayer K (1999). Simulation of scanning laser technique for optical imaging o blood-related intrinsic signals. J Opt Soc Am A 16:58-70.

Stetter M, Schiessl I, Otto T, Sengpiel F, Hubener M, Bonhoeffer T, Obermayer K (2000). Principal component analysis and blind separation of sources for optical imaging of intrinsic signals. Neuroimage 11:482-90.

Swindale NV, Matsubara JA, Cynader MS (1987). Surface organization of orientation and direction selectivity in cat area 18. J Neurosci 7:1414-27.

Thulborn KR, Waterton JC, Mattews PM, Radda GK (1982). Dependence of the transverse relaxation time of water protons in whole blood at high field. Biochem Biophys Acta, 714.

Tootell RB, Mendola JD, Hadjikhani NK, Ledden PJ, Liu AK, Reppas JB, Sereno MI, Dale AM (1997). Functional analysis of V3A and related areas in human visual cortex. J Neurosci 17:7060-78.

Toth LJ, Rao SC, Kim DS, Somers D, Sur M (1996). Subthreshold facilitation and suppression in primary visual cortex revealed by intrinsic signal imaging. Proc Natl Acad Sci USA 93:9869-74.

Toth LJ, Ronen I, Olman C, Merkle H, Ugurbil K, Kim DS (2001). Spatial correlation of BOLD activity with neuronal responses. Soc Neurosci Abstr, pp. 783.1.

Vanzetta I, Grinvald A (1999). Increased cortical oxidative metabolism due to sensory stimulation: implications for functional brain imaging. Science 286:1555-8.

Vanzetta I, Grinvald A (2001). Evidence and lack of evidence for the initial dip in the anesthetized rat: implications for human functional brain imaging. Neuroimage 13:959-67.

Wagner AD, Schacter DL, Rotte M, Koutstaal W, Maril A, Dale A M, Rosen BR, BucknerRL (1998). Building memories: remembering and forgetting of verbal experiences as predicted by brain activity. Science 281:1188-91.

Waldvogel D, van Gelderen P, Muellbacher W, Ziemann U, Immisch I, Hallett M (2000). The relative metabolic demand of inhibition and excitation. Nature 406:995-8.

Yae H, Elias SA, Ebner TJ (1992). Deblurring of 3dimensional patterns of evoked rat cerebellar cortical activity: a study using voltage-sensitive dyes and optical sectioning. J Neurosci Methods 42:195-209. 
